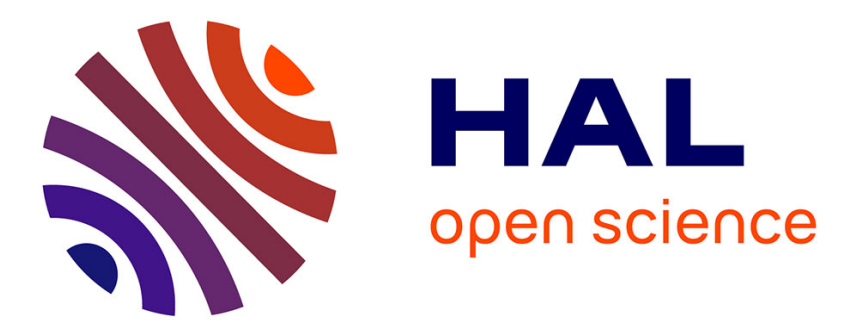

\title{
Toward the use of Symbolic Monte Carlo for Conduction-Radiation Coupling in Complex Geometries
}

Léa Penazzi, Stéphane Blanco, Cyril Caliot, C. Coustet, Mouna El-Hafi, Richard A Fournier, Mathieu Galtier, Loris Ibarrart, Maxime Roger

\section{To cite this version:}

Léa Penazzi, Stéphane Blanco, Cyril Caliot, C. Coustet, Mouna El-Hafi, et al.. Toward the use of Symbolic Monte Carlo for Conduction-Radiation Coupling in Complex Geometries. RAD-19 - 9th International Symposium on Radiative Transfer, Jun 2019, Athens, Greece. 8 p., 10.1615/RAD19.380 . hal-02265075

\section{HAL Id: hal-02265075 https://imt-mines-albi.hal.science/hal-02265075}

Submitted on 22 Nov 2019

HAL is a multi-disciplinary open access archive for the deposit and dissemination of scientific research documents, whether they are published or not. The documents may come from teaching and research institutions in France or abroad, or from public or private research centers.
L'archive ouverte pluridisciplinaire HAL, est destinée au dépôt et à la diffusion de documents scientifiques de niveau recherche, publiés ou non, émanant des établissements d'enseignement et de recherche français ou étrangers, des laboratoires publics ou privés. 


\title{
TOWARD THE USE OF SYMBOLIC MONTE CARLO FOR CONDUCTION-RADIATION COUPLING IN COMPLEX GEOMETRIES
}

\author{
L. Penazzi, ${ }^{1, * 3, *}$ S. Blanco, ${ }^{2}$ C. Caliot,${ }^{3}$ C. Coustet,${ }^{4}$ M. El Hafi, ${ }^{1}$ R. Fournier,${ }^{2}$ M. Galtier, ${ }^{5}$ L. \\ Ibarrart, ${ }^{1,2}$ M. Roger ${ }^{5}$ \\ 1 RAPSODEE, UMR CNRS 5302, IMT Mines Albi, Campus Jarlard, Albi, France \\ 2 LAPLACE, UMR CNRS 5213, Université Paul Sabatier, 118 Route de Narbonne - 31062 \\ Toulouse, France \\ 3 PROMES-CNRS, UPR 851, 7 rue du Four Solaire, 66120 Font Romeu Odeillo, France \\ 4 Méso-Star - 8 rue des Pêchers, 31410 Longages \\ 5 Univ Lyon, CNRS, INSA-Lyon, Université Claude Bernard Lyon 1, CETHIL UMR5008, F-69621, \\ Villeurbanne, France
}

\begin{abstract}
We address the interest of using Symbolic Monte Carlo to obtain a reduced model for conduction-radiation coupling in complex geometries. Symbolic Monte Carlo was successfully used for radiative transfer in a decoupled manner, but no attempt has yet been reported to extend its use to radiation coupled with other modes. Here we show that from a unique Monte Carlo simulation of radiation coupled with conduction in a semi-transparent solid surrounded by a convective flow, it is possible to build a formulation of the local temperature as function of the convective heat transfer coefficient, for instance, including the evaluation of uncertainty. This reduced model (a transfer function) enables to decrease the computation time when the function needs to be evaluated plenty of times for different values of the parameters as in optimization or control algorithms.
\end{abstract}

\section{INTRODUCTION}

Radiative transfer simulations may require the use of Monte Carlo method when handling large geometric models and when radiative properties have strong spectral, spatial and directional dependencies. Numerous research efforts have been reported, nearly since the origin of the Monte Carlo method, attempting to extend this handling of physically and geometrically highly refined objects to other heat transfer modes [1,2]. Todays specifically, numerous theoretical developments are being proposed along this line $[3,4]$ trying to address the geometrical refinement of multiscale engineering requirements. Among these theoretical attempts, Fournier et al. [5] proposed a statistical formulation starting from Green formalism that allows the extension to combined heat transfer of the computer graphics techniques used for rendering images of complex scenes. This thermal Monte Carlo method (involving a linearization with temperature of radiative transfer) was applied and validated numerically for combined heat-transfer in porous media by Caliot et al. [6] and Ibarrart et al. [7]. The authors of the present article are also intensively using this approach for engineering applications in contexts where the linearization of radiation is meaningful, e.g. thermal control of building, urban climate, cooling of electronics, or cooling of electric motors. The objective of the present communication is to show that such calculations can produce not only a computation result but also a transfer function, i.e. a fast reduced version of the model that can be used instead of the full Monte Carlo 
computation when numerous repetitions of the computation are required (command, optimization, inversion).

We achieve this objective using the Symbolic Monte Carlo (SMC) approach [8]. Originally, the SMC method was introduced as the "Inverse Monte Carlo method" [9] and developed for inverse problems applied to radiative transfer problems [10]. Lately, introducing null collisions to handle the nonlinearity of Beer extinction [11,12], the SMC method was developed to express radiative observables as symbolic functions of absorption and scattering coefficients [13]. To the best of our knowledge, this is here the first reported attempt to make use of SMC for combined heat transfer.

The present discussion is essentially theoretical and not applicative. We stick to simplified stationary physics in order to help clarifying the formal developments. We leave aside spectral dependences and heterogeneities that are already recognized strengths of Monte Carlo approaches and only concentrate on proving that i) SMC can be extended to combined heat-transfer algorithms; ii) it preserves the features of Monte Carlo approaches as far as handling large geometric models is concerned. We retain the case of grey radiation coupled with conduction in a homogeneous semi-transparent solid surrounded by a convective flow, using a uniform heat transfer coefficient $h$ and a uniform fluid temperature $T_{f}$. We formulate the local solid temperature as a function of $h$, together with its uncertainty, on a parameter range that widely extends the validity range of first order Taylor extensions, i.e. proving that SMC transfer functions accurately capture the physical nonlinear response to $h$ variations. The grey and homogeneity assumptions could easily be suppressed, but this is not the case for one major assumption that todays limits the application potential to the above mentioned fields: the temperature differences viewed by the transfer of radiation (between the emission and absorption locations for a given path) must be small enough for the Planck function to be linearized as function of temperature. We discuss simulation results using reference examples of the Large Geometric Model Archive (https://www.cc.gatech.edu/projects/large_models/) initiated by the computer graphics community [14].

\section{THE SYMBOLIC MONTE CARLO MODEL}

The heat transfer model The studied configuration (Figure 2) is composed of a semi-transparent solid domain $\mathcal{D}_{S}$ and a transparent fluid domain $\mathcal{D}_{F}$. Coupled conduction and radiation are considered in the solid, convection at the solid/fluid interface $\partial \mathcal{D}_{S}$ and radiation in the fluid. Let us name $\vec{x}$ a location inside the solid, $\vec{y}$ a location at the solid/fluid interface and $\vec{n} \equiv \vec{n}(\vec{y})$ the unit normal to the interface at $\vec{y}$, heading toward the fluid. The solid temperature at $\vec{x}$ is noted $T \equiv T(\vec{x})$. The solid properties are homogeneous and independent of temperature: $\lambda$ is the thermal conductivity, $k_{a}$ the absorption coefficient and $k_{s}$ the scattering coefficient. The fluid is assumed perfectly mixed at a known temperature $T_{f}$ : the boundary layer is only represented using a convective heat transfer coefficient $h$ at the solid/fluid interface (equation (3)). Incoming radiation at the boundary of this system is fixed by assuming a black bounding box $(\partial \Omega)$ at temperature $T_{\infty}$. We use the following simple heat-transfer model (linear in temperature):

$$
\left\{\begin{array}{lr}
\lambda \Delta T+\zeta\left(T_{R}-T\right)=0 & \forall \vec{x} \in \mathcal{D}_{S} \\
T_{R}=\int_{\mathcal{D}_{\Gamma}} p_{\Gamma}(\gamma) d \gamma T\left(\vec{x}_{\gamma}\right) & \forall \vec{x} \in \mathcal{D}_{S} \\
-\lambda \vec{\nabla} T \cdot \vec{n}=h\left(T-T_{f}\right) & \forall \vec{x} \equiv \vec{y} \in \partial \mathcal{D}_{S} \\
T=T_{f} & \forall \vec{x} \in \mathcal{D}_{F}
\end{array}\right.
$$


In this model, radiative heat transfer is steady and linearized around a reference temperature $T_{\text {ref }}$, using a volumic heat transfer coefficient $\zeta=16 k_{a} \sigma T_{r e f}^{3}$ in which $\sigma$ is the Stefan-Boltzmann constant. $T_{R}(\vec{x})$ is defined using the space $\mathcal{D}_{\Gamma}$ of all the radiative-paths $\gamma$, of probability density $p_{\Gamma}(\gamma)$, corresponding to the statistics of photons emitted at $\vec{x}$ until their absorption at $\vec{x}_{\gamma}$ : this radiative temperature is simply the mean temperature at absorption locations, $T_{R}(\vec{x})=<T_{R}(\vec{x})_{\gamma}>_{\mathcal{D}_{\Gamma}}$.

The equivalent statistical model The heat transfer equation (1) and the solid/fluid boundary condition (3) are reformulated to get a statistical interpretation allowing a Monte Carlo approach. The application of Monte Carlo Methods to evaluate solutions of heat equation received much early attention $[15,16]$. But even today, work continues on developing new Monte Carlo algorithms for specific applications [17,18]. Along this line, if we consider the term in $T_{R}$ as a source for equation (1), we can recognize the classical Helmholtz equation. As shown by Mikhailov [18], this equation can be solved with an exact scheme inside the domain by the walking-on-spheres algorithm. However, the implementation can be tricky in the general case. For practical reasons, we choose here an approximate scheme built from the assumption that the radiative term $\zeta\left(T_{R}-T\right)$ is quasi-constant inside spheres of small radius $\delta$, which gives the classical solution resulting from reformulating the Poisson equation with constant source $[1,8]$ :

$$
T(\vec{x}) \approx \frac{1}{4 \pi} \int_{4 \pi} T(\vec{x}+\delta \vec{u}) d \vec{u}+Q(\delta)
$$

with $\vec{u}$ integrated over the unit sphere, $Q(\delta)=q_{0} \frac{\delta^{2}}{6}$ and $q_{0}=\frac{\zeta}{\lambda}\left(T_{R}-T(\vec{x})\right)$. The first term comes from the classical result of harmonics functions theory (i.e. without sources, the average value of $T$ over the surface of a sphere of any radius $\delta$, equals the value of $T$ at the center of the sphere). The boundary condition of equation (3) is also discretized with a backward difference scheme to get (6):

$$
\vec{\nabla} T \cdot \vec{n} \approx \frac{T(\vec{y})-T(\vec{y}-\delta \vec{n})}{\delta}
$$

In equations (5) and (6), $\delta$ is a numerical parameters: the walking step for conduction in the solid. In equation (5), when $\vec{x}$ is in the vicinity of the boundary, $\delta$ inside the angular integral must be adjusted and numerous strategies are available in the literature [16]. Here we replace $\delta$ with $\delta_{c} \equiv \delta_{c}(\vec{x}, \vec{u})$ defined the following way. If the segment $[\vec{x}-\delta \vec{u}, \vec{x}+\delta \vec{u}]$ does not intersect the boundary, then $\delta_{c}$ is kept equal to $\delta$. Otherwise, $\vec{y}$ is defined as the intersection the closest to $\vec{x}$ and $\delta_{c}=\|\vec{x}-\vec{y}\|$. Similarly, $\delta$ is also adjusted in equation (6) in order to avoid that $\vec{y}-\delta \vec{n}$ be outside the solid for high-frequency boundary structures. $\delta$ is replaced with $\delta_{b} \equiv \delta_{b}(\vec{y}, \vec{n})$ defined the following way. If the segment $[\vec{y}, \vec{y}-\delta \vec{n}]$ does not intersect the boundary, then $\delta_{b}$ is kept equal to $\delta$. Otherwise, $\overrightarrow{y^{\prime}}$ is defined as the intersection the closest to $\vec{y}$ and $\delta_{b}=\frac{1}{2}\left\|\vec{y}-\overrightarrow{y^{\prime}}\right\|$.

By using these $\delta$ adjustments and introducing expressions (5) and (6) into equations (1) and (3), the temperature in the solid $T(\vec{x})$ and at the boundary $T(\vec{y})$ can be formulated as:

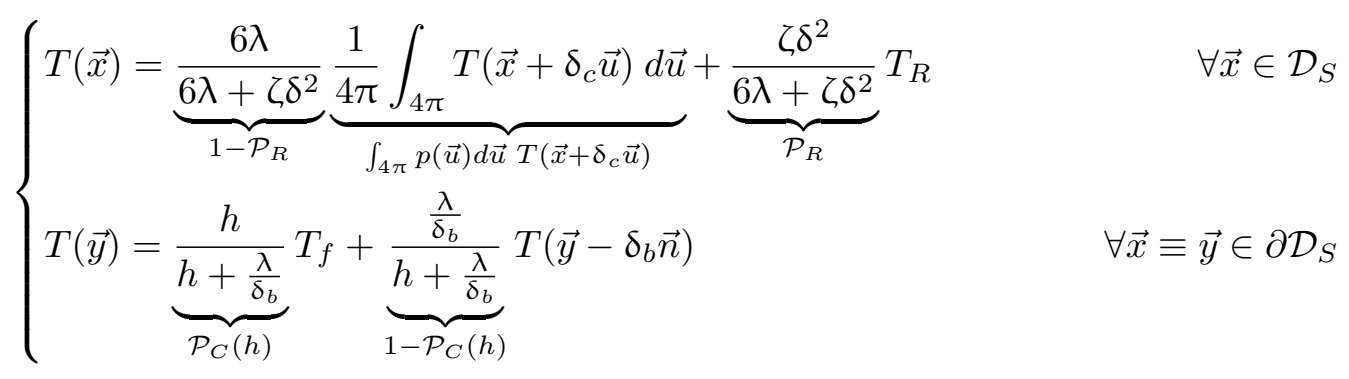

Equations (7) and (8) can be given a statistical interpretation by introducing the probability $\mathcal{P}_{R}=$ $\frac{\zeta \delta^{2}}{6 \lambda+\zeta \delta^{2}}$, the probability $\mathcal{P}_{C}(h)=\frac{h}{h+\frac{\lambda}{\delta_{b}}}$, and the probability density $p(\vec{u})=\frac{1}{4 \pi}$, and can be gathered 
using the Heaviside function $\mathcal{H}$ (taking the value 1 if the condition is satisfied and 0 otherwise),

$$
\begin{aligned}
T(\vec{x}) & =\mathcal{H}\left(\vec{x} \in \mathcal{D}_{S}\right) \times\left\{\left(1-\mathcal{P}_{R}\right) \times \int_{4 \pi} p(\vec{u}) d \vec{u} T\left(\vec{x}+\delta_{c} \vec{u}\right)+\mathcal{P}_{R} \times T_{R}\right\} \\
& +\mathcal{H}\left(\vec{x} \equiv \vec{y} \in \partial \mathcal{D}_{S}\right) \times\left\{\mathcal{P}_{C} \times T_{f}+\left(1-\mathcal{P}_{C}\right) \times T\left(\vec{y}-\delta_{b} \vec{n}\right)\right\}
\end{aligned}
$$

in order to justify theoretically the Monte Carlo algorithm of Figure 1. This allows indeed to look at $T(\vec{x})$ as the expectation of a random variable $W$ (the Monte Carlo weight) associated to a conductoradiative path and taking either the value $T_{f}$ if the path ends with a convective exchange at the solid/fluid interface, or the value $T_{\infty}$ if the path ends with radiation at the bounding box [5,19]. $N$ paths are sampled, providing $N$ realisations $w_{1}, w_{2} \ldots w_{N}$ of $W$, and $T(\vec{x})$ is estimated as $T(\vec{x}) \approx$ $\frac{1}{N} \sum_{i=1}^{N} w_{i}$. The heart of the path-sampling of Figure 1 is the following. The path starts at $\vec{x}$ within the solid by first choosing between a conductive or a radiative step. If a radiative step is chosen, then the algorithm is a very standard Monte Carlo algorithm for radiation in multiple scattering media and ends at location $\vec{z}$ either with an absorption inside the solid, or with absorption at the bounding box. If absorption occurs at the bounding box, the path stops and the Monte Carlo weight is $\omega=T_{\infty}$. If absorption occurs inside the solid, this defines a new $\vec{x}$ location and the path sampling algorithm loops at its start. When a conductive step is chosen, the step direction $\vec{u}$ is sampled isotropically and $\delta_{c}$ is computed by testing the intersections with the boundary when travelling from $\vec{x}$ in direction $\vec{u}$ and direction $-\vec{u}$. Once the conductive step has been made, it ends either within the solid or at the solid/fluid interface. If the conductive step ends within the solid, this defines a new $\vec{x}$ location and the path sampling algorithm loops at its start. If the conductive step ends at a location $\vec{y}$ at the solid/fluid interface, $\delta_{b}$ is computed by testing the intersections with the boundary when travelling from $\vec{y}$ in direction $-\vec{n}$. Once $\delta_{b}$ has been computed, a Bernoulli test is made to decide wether the path continues from the interface with conduction or with convection. If convection is chosen, the path stops and the Monte Carlo weight is $\omega=T_{f}$. If conduction is chosen, $\vec{x}$ is set to the location $\vec{y}-\delta_{b} \vec{n}$ and the path sampling algorithm loops at its start.

The corresponding Symbolic model Now that we have defined the Monte Carlo algorithm evaluating $T(\vec{x}, h)$ for a given value of $h$ (let say $h=h_{r e f}$ ), we can address the true objective of the present paper and implement a symbolic Monte Carlo approach in order to produce a reduced model of how $T(\vec{x}, h)$ varies with $h$. This could be achieved partially by computing the sensitivity $\left.\frac{\partial T}{\partial h}\right|_{h=h_{r e f}}$ [20,21] and using a first order Taylor expansion, but then the ability to predict $T(\vec{x})$ as a function of $h$ would be limited to its linearity range around $h_{r e f}$. The whole interest of the symbolic approach is that it extends this limit and captures nonlinear responses.

The starting point is to rewrite the solid/fluid boundary equation (8) using an importance sampling approach, i.e. introducing a new arbitrary probability $\tilde{\mathcal{P}}=\frac{h_{r e f}}{h_{r e f}+\frac{\lambda}{\delta_{b}}}$ and correcting the Monte Carlo weights accordingly:

$$
T(\vec{y}, h)=\tilde{\mathcal{P}}\left\{\frac{\mathcal{P}_{C}(h)}{\tilde{\mathcal{P}}} T_{f}\right\}+(1-\tilde{\mathcal{P}})\left\{\frac{1-\mathcal{P}_{C}(h)}{1-\tilde{\mathcal{P}}} T\left(\vec{y}-\delta_{b} \vec{n}\right)\right\}
$$

Let us first consider the simple case of a sphere (Figure 2): as there is no risk of intersection with the geometry during re-injection, $\delta_{b}$ has the same value at each location $\vec{y}$ on the boundary. This means that the Monte Carlo algorithm built from Equation (10) instead of Equation (8) is strictly identical to that of Figure 1, except that when reaching the boundary the uniformly random variable $R$ is compared to $\tilde{\mathcal{P}}$ instead of $\mathcal{P}_{C}(h)$, and the Monte Carlo weight $w$ is multiplied by $\frac{\mathcal{P}_{C}(h)}{\tilde{\mathcal{P}}}$ or by $\frac{\left(1-\mathcal{P}_{C}(h)\right)}{1-\tilde{\mathcal{P}}}$, depending on the comparison. As a result of this quite simple transformation, the path statistics do not depend on $h$ anymore: the same sampled paths can be used to evaluate $T(\vec{x}, h)$ for any value of $h$ because $h$ appears in the Monte Carlo weight only. The symbolic formulation is then constructed by simply storing these weights in their $h$ dependance. For the $i$-th path, let us 


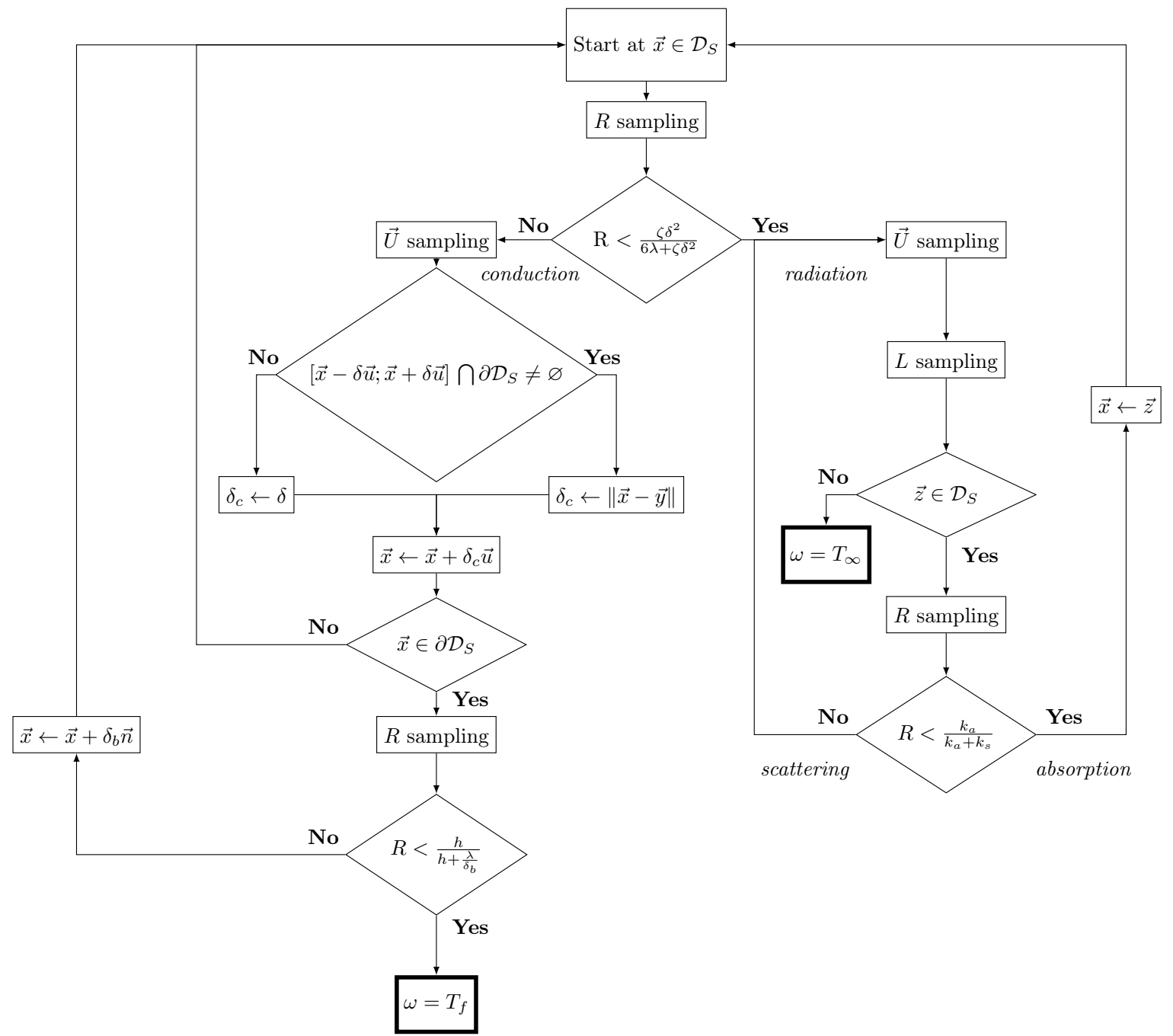

Figure 1. The path-sampling algorithm. The random variable $\mathrm{R}$ is uniformly distributed on the unit interval.

note $j_{i}$ the number of times the path has reached the boundary and was re-injected in the solid. The corresponding Monte Carlo weight $w_{i}(h)$ is therefore

$$
\begin{array}{ll}
\omega(h)_{i}=\left(\frac{1-\mathcal{P}_{C}(h)}{1-\tilde{\mathcal{P}}}\right)^{j_{i}} T_{\infty} & \text { if the path ends with a radiative path } \\
\omega(h)_{i}=\left(\frac{1-\mathcal{P}_{C}(h)}{1-\tilde{\mathcal{P}}}\right)^{j} \frac{\mathcal{P}_{C}(h)}{\tilde{\mathcal{P}}} T_{f} & \text { if the path ends with a convective path }
\end{array}
$$

For generalization to any geometry, we need to deal with the fact that $\delta_{b}$ has different values at each location $\vec{y}$ on the boundary. $\mathcal{P}_{C}(h)$ is therefore not a unique function of $h$. We note $\mathcal{P}_{C, i, m}(h)$ the particular function for the $m$-th boundary encounter in the $i$-th path, i.e. $\mathcal{P}_{C, i, m}(h)=\frac{h}{h+\frac{\lambda}{\delta_{b, i, m}}}$ were $\delta_{b, i, m}$ is the re-injection distance at the corresponding boundary location. With this notation, the Monte Carlo weights is

$$
\begin{aligned}
& \omega(h)_{i}=\left(\prod_{m=1}^{j_{i}} \frac{1-\mathcal{P}_{C, i, m}(h)}{1-\tilde{\mathcal{P}}}\right) T_{\infty} \quad \text { if the path ends with a radiative path } \\
& \omega(h)_{i}=\left(\prod_{m=1}^{j_{i}} \frac{1-\mathcal{P}_{C, i, m}(h)}{1-\tilde{\mathcal{P}}}\right) \frac{\mathcal{P}_{C, i, j_{i}}(h)}{\tilde{\mathcal{P}}} T_{f} \quad \text { if the path ends with a convective path }
\end{aligned}
$$

The estimate of $T(\vec{x}, h)$ and the estimate of its standard deviation are then constructed using the 
Monte Carlo weights as in any Monte Carlo approach:

$$
\begin{aligned}
& T(\vec{x}, h) \simeq \frac{1}{N} \sum_{i=1}^{N} \omega(h)_{i} \\
& \sigma(h)=\frac{1}{\sqrt{N}}\left[\frac{1}{N} \sum_{i=1}^{N} \omega^{2}(h)_{i}-\left(\frac{1}{N} \sum_{i=1}^{N} \omega(h)_{i}\right)^{2}\right]^{\frac{1}{2}}
\end{aligned}
$$

Table 1 illustrates the typical storage procedure, using the path examples of Figure 2, for both the sphere case and the generalized case. In Figure 3, full SMC results are presented and compared with the reference Monte Carlo simulations. The statistical uncertainties, displayed as error bars (the standard deviation of the estimator), confirm the theoretical predictions: whatever the value of $h$, even far from $h_{r e f}$, the predicted accuracy of the SMC calculation can be trusted (provided that the accuracy of the initial Monte Carlo could already be trusted, i.e. no pathological rare-events statistics were encountered). Again, the SMC formulation preserves here the features of the initial Monte Carlo. As expected, when $h$ is far from $h_{r e f}$, the error bar increases. But the prediction remains quite accurate way outside the linearity zone (the range of $h$ for which $T(\vec{x})$ depends on $h$ linearly). In the present example, the SMC calculation can therefore be faithfully used instead of the complete Monte Carlo run for all control, inversion or optimum-design procedures requiring large numbers of iterative calls. It is very hard to predict how much this conclusion extends to other parameters than $h$ and other heat transfer configurations than the present conductive-radiative one, but the feasibility is established. We have repeated the same simulations for other large geometric models, increasing the number of triangles of two orders of magnitude and the conclusions are identical, with nearly identical computation times (up to the limit of the available memory).

Table 1: Examples of the SMC storage requirements for the path examples of Figure 2

\begin{tabular}{|c|c|c|c|cc|}
\hline Realization & Path & MC $w$ & \multicolumn{2}{|c|}{ MC $w(h)$} \\
\hline & & & Sphere & Generalization \\
\hline $\mathrm{i}=1$ & $A \rightarrow B_{1}$ & $T_{f}$ & $\left(\frac{1-\mathcal{P}_{C}(h)}{1-\tilde{\mathcal{P}}}\right)^{2} \times \frac{\mathcal{P}_{C}(h)}{\tilde{\mathcal{P}}} T_{f}$ & $\left(\frac{1-\mathcal{P}_{c_{1}}(h)}{1-\tilde{\mathcal{P}}_{1}}\right) \times\left(\frac{1-\mathcal{P}_{c_{2}}(h)}{1-\tilde{\mathcal{P}}_{2}}\right) \times \frac{\mathcal{P}_{c_{3}}(h)}{\tilde{\mathcal{P}}_{3}} T_{f}$ \\
$\mathrm{i}=2$ & $A \rightarrow B_{2}$ & $T_{\infty}$ & $\left(\frac{1-\mathcal{P}_{C}(h)}{1-\tilde{\mathcal{P}}}\right)^{2} \times T_{\infty}$ & $\left(\frac{1-\mathcal{P}_{c_{1}}(h)}{1-\tilde{\mathcal{P}}_{1}}\right) \times\left(\frac{1-\mathcal{P}_{c_{2}}(h)}{1-\tilde{\mathcal{P}}_{2}}\right) \times \frac{\mathcal{P}_{c_{3}}(h)}{\tilde{\mathcal{P}}_{3}} T_{\infty}$ \\
$\mathrm{i}=\mathrm{N}$ & $A \rightarrow B_{N}$ & $T_{f}$ & $\left(\frac{1-\mathcal{P}_{C}(h)}{1-\tilde{\mathcal{P}}}\right)^{3} \times \frac{\mathcal{P}_{C}(h)}{\tilde{\mathcal{P}}} T_{f}$ & $\left(\frac{1-\mathcal{P}_{c_{1}}(h)}{1-\tilde{\mathcal{P}}_{1}}\right) \times\left(\frac{1-\mathcal{P}_{c_{2}}(h)}{1-\tilde{\mathcal{P}}_{2}}\right) \times\left(\frac{1-\mathcal{P}_{c_{3}}(h)}{1-\tilde{\mathcal{P}}_{3}}\right) \times \frac{\mathcal{P}_{c_{4}}(h)}{\tilde{\mathcal{P}}_{4}} T_{f}$ \\
\hline
\end{tabular}

\section{CONCLUSION}

The present discussion still remains far from realistic industrial configurations. But producing fast transfer functions for large geometric models and evaluating their accuracy level is a deep industrial concern and is worth a close theoretical exploration. We can conclude here that SMC can be an efficient corresponding approach and that it is widely insensitive to the level of the geometric refinement. The next step will be first to suppress the grey medium assumption and the homogeneity assumptions for solid and convective properties. Part of this has already been tested and the remaining should not introduce any specific difficulty: Monte Carlo has already been shown to deal with these physical refinements in a quite straightforward manner. Planck function linearization is a much stronger assumption, but Dauchet et al. have shown how Monte Carlo could address successfully some nonlinear physics using branching processes [12]. The following major step will therefore be to evaluate the potential of such branching statistics in front of the nonlinear temperature dependance of the Planck function and how they fit in the SMC framework. 

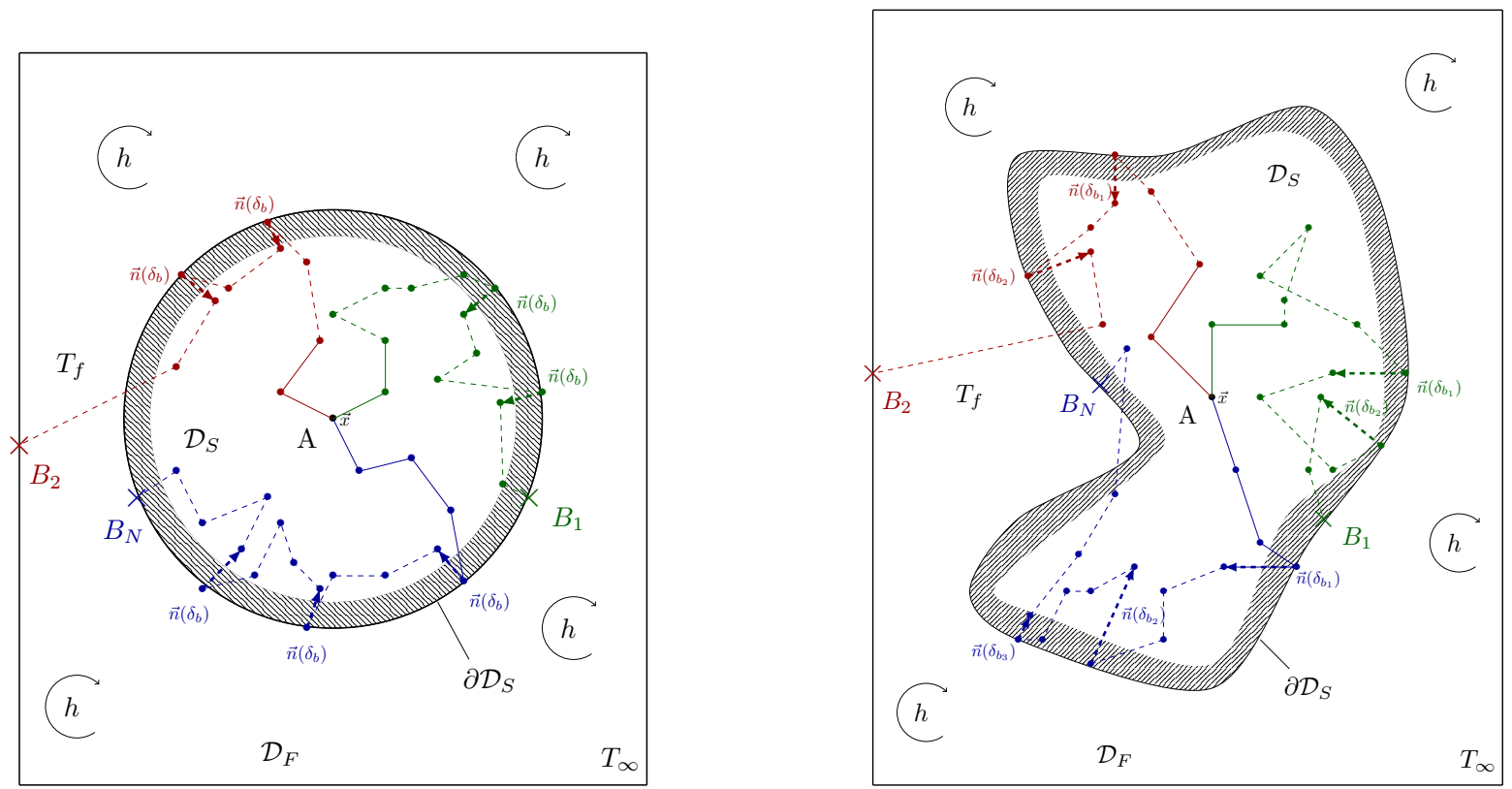

Figure 2. Examples of random paths inside a sphere (left) or any geometry (right). Three paths start at the same location $A$ (the location where the temperature is to be estimated). Each path starts with a conductive step and ends in $B_{1}, B_{2}$ and $B_{N}$ correspondingly to the $1^{\text {st }}, 2^{\text {nd }}$ and $N^{\text {th }}$ realization. Continuous lines represent conductive steps from one point (bullet) to the other, and dashed lines represent radiative steps, either absorption or scattering.
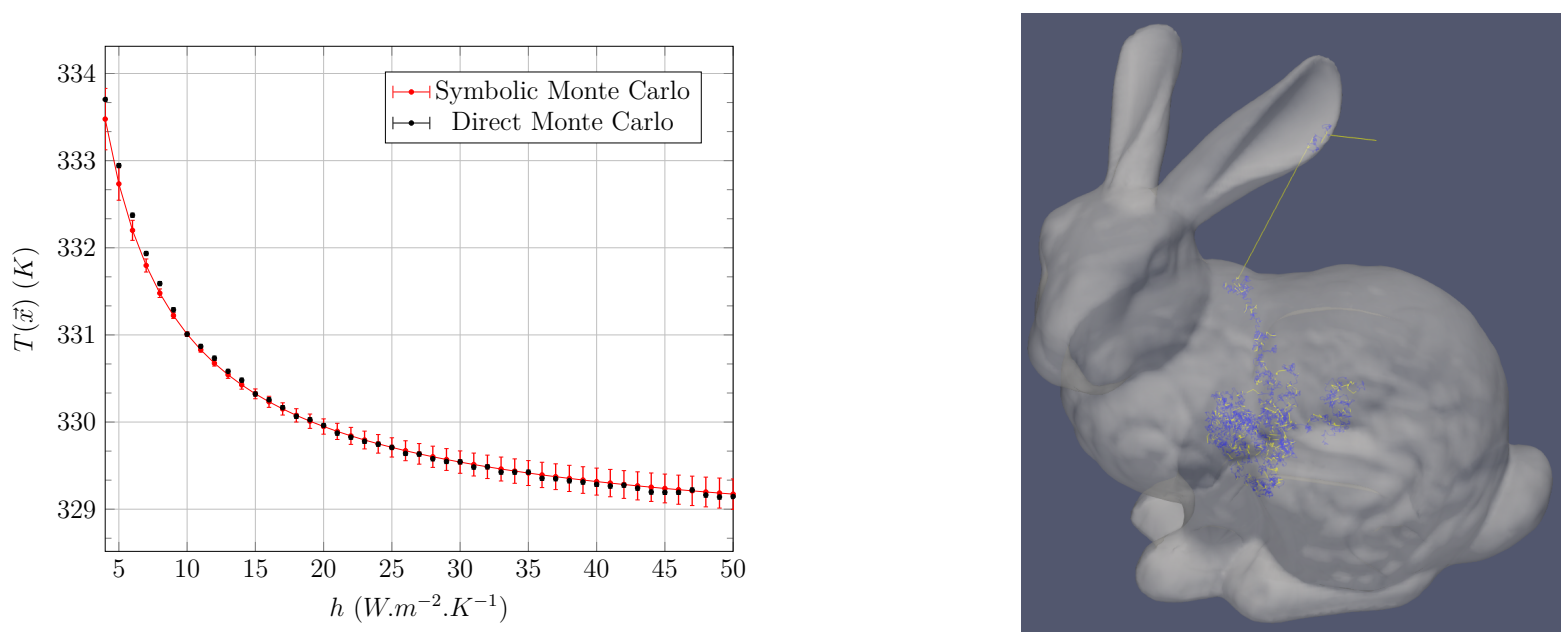

Figure 3. Left - Comparison of the Symbolic Monte Carlo method (red marks, 1' 39") with the Direct Monte Carlo method (black marks, the reference) for evaluation of local temperature as function of the convective heat transfer coefficient (left). The test is made on the Stanford bunny geometry [14]. Its volume and surface are noted $V$ and $S$ respectively and we retain $L=4 V / S(L=1 \mathrm{~m})$ as caracteristic size. The probe location is $\frac{\vec{x}}{L}=(0 ; 0.1 ; 0)$ (in the middle of the solid). The reference temperature is $T_{\text {ref }}=325 \mathrm{~K}$. The fixed physical parameters are $\frac{T_{f}-T_{\text {ref }}}{T_{\text {ref }}}=-0.08\left(T_{f}=300 K\right), \frac{T_{\infty}-T_{\text {ref }}}{T_{\text {ref }}}=+0.08\left(T_{\infty}=350 K\right), k_{a} L=0.1$ (leading to $\left.\zeta=3.11 \mathrm{~W} \cdot \mathrm{m}^{-3} \cdot K^{-1}\right), k_{s} L=0.1, \frac{\lambda}{\zeta L^{2}}=0.1\left(\lambda=0.311 \mathrm{~W} \cdot \mathrm{m}^{-2} \cdot K^{-1}\right), \frac{h_{r e f} L}{\lambda}=16.056\left(h_{r e f}=\right.$ $\left.10 \mathrm{~W} \cdot \mathrm{m}^{-2} \cdot K^{-1}\right), \frac{\delta}{L}=0.025 \mathrm{~m}$ and $N=10^{5}$. The computation of the temperature with the SMC is around 20000 times faster than the computation by a direct MC. Right - One conducto-radiative path exemple.

\section{ACKNOWLEDGMENTS}

This project has received funding from the programme "investissement d'avenir" (investments for the future) of the "Agence Nationale de la Recherche" (National Agency for Research) of the French state under award number "ANR-10-LBX-22-01-SOLSTICE". We acknowledge support from the 
Agence Nationale de la Recherche (ANR, grant HIGH-TUNE ANR-16-CE01-0010, http://www.umrcnrm.fr/high-tune and ANR, grant MGC-RAD) and from Region Occitanie (Projet CLE-2016 EDStar).

\section{REFERENCES}

[1] A. Haji-Sheikh and E. M. Sparrow, "The Solution of Heat Conduction Problems by Probability Methods," Mechanical Engineering, vol. 89, no. 2, p. 10, 1967.

[2] M. N. Ozisik, Boundary Value Problems of Heat Conduction, courier corporation ed., 2013.

[3] G. L. Vignoles and A. Ortona, "Numerical study of effective heat conductivities of foams by coupled conduction and radiation," International Journal of Thermal Sciences, vol. 109, pp. 270-278, 2016.

[4] G. L. Vignoles, "A hybrid random walk method for the simulation of coupled conduction and linearized radiation transfer at local scale in porous media with opaque solid phases," International Journal of Heat and Mass Transfer, vol. 93, pp. 707-719, 2016.

[5] R. Fournier, S. Blanco, V. Eymet, M. E. Hafi, and C. Spiesser, "Radiative, Conductive and Convective HeatTransfers in a Single Monte Carlo Algorithm," Journal of Physics: Conference Series, vol. 676, 2016.

[6] C. Caliot, S. Blanco, C. Coustet, M. El Hafi, V. Eymet, V. Forest, R. Fournier, and B. Piaud, "Combined conductiveradiative heat transfer analysis in complex geometry using the monte carlo method," Eurotherm Seminar 110 Computational Thermal Radiation in Participating Media - VI, 2015.

[7] L. Ibarrart, S. Blanco, R. Fournier, and M. ElHafi, "Combined conductive-convective-radiative heat transfer in complex geometry using the Monte Carlo method," in The 16th International Heat Transfer Conference - Chinese National Convention Center, Beijing, China, August 10-15, 2018.

[8] W. L. Dunn and J. K. Shultis, Exploring Monte Carlo Methods, elsevier ed., 2012.

[9] W. L. Dunn, "Inverse Monte Carlo Analysis," Journal of Computational Physics, vol. 41, no. 1, pp. 154-166, 1981.

[10] S. Subramaniam and M. P. Mengüç, "Solution of the Inverse Radiation Problem for Inhomogeneous and Anisotropically Scattering Media Using a Monte Carlo Technique,” International journal of heat and mass transfer, vol. 34, no. 1, pp. 253-266, 1991.

[11] M. Galtier, S. Blanco, C. Caliot, C. Coustet, J. Dauchet, M. El Hafi, V. Eymet, R. Fournier, J. Gautrais, A. Khuong, B. Piaud, and G. Terrée, "Integral formulation of null-collision Monte Carlo algorithms," Journal of Quantitative Spectroscopy and Radiative Transfer, vol. 125, pp. 57-68, Apr., 2013.

[12] J. Dauchet, J.-J. Bezian, S. Blanco, C. Caliot, J. Charon, C. Coustet, M. El Hafi, V. Eymet, O. Farges, V. Forest, R. Fournier, M. Galtier, J. Gautrais, A. Khuong, L. Pelissier, B. Piaud, M. Roger, G. Terrée, and S. Weitz, “Addressing Nonlinearities in Monte Carlo," Scientific Reports, vol. 8, no. 1, 2018.

[13] M. Galtier, M. Roger, F. André, and A. Delmas, "A Symbolic Approach for the Identification of Radiative Properties," Journal of Quantitative Spectroscopy and Radiative Transfer, vol. 196, pp. 130-141, 2017.

[14] G. Turk and M. Levoy, "Zippered Polygon Meshes from Range Images," in Proceedings of the 21st Annual Conference on Computer Graphics and Interactive Techniques - SIGGRAPH '94, ACM Press, pp. 311-318, 1994.

[15] J. H. Curtiss, "Sampling methods applied to differential and difference equations with special reference to equations of the elliptic type," in Bulletin of the American Mathematical Society, vol. 57, Amer Mathematical Soc 201 Charles St, Providence, RI 02940-2213, pp. 73-73, 1951.

[16] M. E. Muller, "Some Continuous Monte Carlo Methods for the Dirichlet Problem," 1956.

[17] C.-O. Hwang and M. Mascagni, "Efficient modified "walk on spheres" algorithm for the linearized PoissonBolzmann equation," Applied Physics Letters, vol. 78, no. 6, pp. 787-789, 2001.

[18] G. A. Michajlov, New Monte Carlo methods with estimating derivatives, VSP, 1995.

[19] M. Kac, "Random Walk and the Theory of Brownian Motion," p. 24, 1947.

[20] A. de Lataillade, S. Blanco, Y. Clergent, J. L. Dufresne, A. El Hafi, and R. Fournier, "Monte Carlo method and sensitivity estimations," Journal of Quantitative Spectroscopy \& Radiative Transfer, vol. 75, no. 5, pp. 529-538, 2002. 13.

[21] M. Roger, S. Blanco, M. El Hafi, and R. Fournier, "Monte Carlo estimates of domain-deformation sensitivities," Physical review letters, vol. 95, no. 18, p. 180601, 2005. 